\title{
RATIONAL CURVE WITH ONE CUSP. II
}

\author{
HISAO YOSHIHARA
}

\begin{abstract}
Let $C$ be a plane curve with $C-\{P\} \cong \mathbf{A}^{1}$ for some point $P \in C$ and degree $d \geq 3$. Let $\left\{e_{1}, \ldots, e_{t}\right\}$ be the multiplicities of the infinitely near
\end{abstract} singular points of $P$. Then the following three conditions are equivalent:

(1) $C \backslash L \cong \mathbf{A}^{1}$ for some line $L$,

(2) $d=e_{1}+e_{2}$ (in case $t=1$, let $e_{2}=1$ ),

(3) $R=d^{2}-\sum_{i=1}^{t} e_{i}^{2}-e_{t}+1 \geq 3$.

1. This paper is a continuation of [2]. We shall give an affirmative answer to the problem raised in that paper, which is a numerical criterion for a curve of type I to be type II. By the criterion the relation between types I and II will be clear. We follow the notation and convention used in [2].

THEOREM. Suppose that $C$ is a curve of type I and $d \geq 3$. Then $C$ is of type II if and only if $R \geq 3$.

Recalling the result [2, Lemma 1], we see that conditions (1) and (2) in the abstract are equivalent. In case $d=1$ and 2 , since $t=0$, we put $R=2$ and 5 respectively. Then we obtain the following from the lemma in the next section.

COROLlaRY. Suppose that $C$ is a curve of type I. Then it is a line if and only if $R=2$.

2. We shall prove the next lemma. Then the proof of the Theorem will be complete, since the only if part has been proved [3, Lemma 5.1].

LEMMA. If $C$ is a curve of type I with $R \geq 2$, then it is of type II.

PrOOF. First we fix some notation. Let

$$
\mathbf{P}^{2}=S_{0} \stackrel{\sigma_{1}}{\longleftarrow} S_{1} \stackrel{\sigma_{2}}{\longleftarrow} \cdots \stackrel{\sigma_{k-1}}{\longleftarrow} S_{k-1} \stackrel{\sigma_{k}}{\longleftarrow} S_{k}
$$

be a sequence of blow-ups $\sigma_{i}$ with successive centers $P_{i}$ in $S_{i-1}$ and put $\sigma=$ $\sigma_{1} \cdots \sigma_{k}$. For a birational transformation $g$ we denote by $g(X)$ and $g[X]$ the total and proper transforms of $X$ respectively. Then put $E_{i}=\sigma_{i}^{-1}\left(P_{i}\right)$ and $E_{i}^{\prime}=$ $\left(\sigma_{i+1} \cdots \sigma_{k}\right)^{-1}\left[E_{i}\right]$, where $1 \leq i \leq k-1$. For a curve $X$ on a surface, let $X^{2}$ denote the self-intersection number of $X$ on the surface. Let $k$ be minimal in order that $\sigma^{-1}(C)$ has only simple normal crossings and $C^{2}=0$, where $C^{\prime}=\sigma^{-1}[C]$. This is possible, since $R \geq 2$. Then we have $k=t+e_{t}+R-1$. Since $C^{\prime}$ is a nonsingular rational curve with $C^{\prime 2}=0$, there is a morphism $f: S=S_{k} \rightarrow \mathbf{P}^{1}$ defined by the linear system $\left|C^{\prime}\right|[\mathbf{1}]$. Putting $\mathcal{E}=E_{1}^{\prime}+\cdots+E_{k-1}^{\prime}$, we have that $\mathcal{E} \cap C^{\prime}=\varnothing$. Since $R \geq 2, \mathcal{E}$ is connected and hence it is contained in a single fiber $₹$. By the above definition we see that $E_{k}$ is a section of $f$ and $E_{k}^{2}=-1$.

Received by the editors May 2, 1986.

1980 Mathematics Subject Classification (1985 Revision). Primary $14 \mathrm{H} 45$. 
Note that ${E_{i}^{\prime}}^{2} \leq-2$ for $i \neq k$. Referring to [1] again, we see that $\mathcal{F}$ contains an exceptional curve of the first kind $\Delta^{\prime}$ and $\mathcal{F}=\mathcal{E}+\Delta^{\prime}$ as a divisor. Comparing the number of blow-ups to obtain $S$ from $\mathbf{P}^{2}$ with that of the irreducible components of $\mathcal{F}$, we infer that $f$ has no singular fiber except $\mathcal{F}$. By contracting successively the components of $\mathcal{F}$, we get a birational morphism $\tau: S \rightarrow F$ and then a morphism $\bar{f}: F \rightarrow \mathbf{P}^{1}$, where $\bar{f}$ has no singular fiber. Here we may assume that $E_{k-1}^{\prime}$ is not contracted by $\tau$. Otherwise $\tau^{-1}$ has fundamental point at $\tau\left(E_{k} \cap \mathcal{F}\right)$. Then by elementary transformation with the center at this point we have a new surface which $S$ dominates, too. We can repeat this procedure to get the surface on which the self-intersection of the image of $E_{k}$ becomes -1 , as was to be shown. Then, by contracting $E_{k}$, we get an isomorphism $\mathbf{P}^{2}-\Delta \cong \mathbf{P}^{2}-L$, where $\Delta=\sigma\left(\Delta^{\prime}\right)$ and $L$ is a line. Since the group $\operatorname{Pic}\left(\mathbf{P}^{2}-\Delta\right)$ is a cyclic group whose order is the degree $\Delta$, the curve $\Delta$ is also a line. On the other hand, by the above definition, we have that $C \cap \Delta=\{P\}$. This means that $C$ is of type II.

\section{REFERENCES}

1. M. H. Gizatullin, On affine surfaces that can be completed by a nonsingular rational curve, Math. USSR-Izv. 4 (1970), 787-810.

2. H. Yoshihara, Rational curve with one cusp, Proc. Amer. Math. Soc. 89 (1983), 24-26.

3. $\_$, On open algebraic surfaces $\mathbf{P}^{2}-C$, Math. Ann. 268 (1984), 43-57.

Faculty of General EduCation, Nigata University, NiIgata 950-21, Japan 